\title{
50 CAN ADVANCE CARE PLANING OFFER AN ALTERNATIVE TO PEOPLE CONTEMPLATING EUTHANASIA?
}

10.1136/bmjspcare-2011-000053.50

R Fullam, A Hancock, K Detering, J Davies, W Silvester Respecting Patient Choices, Austin Health, Melbourne, Australia

Background Many patients with terminal illness and progressive loss of control over their bodies explore the option of euthanasia as a means to regain control. Some of these patients are referred by health professionals for advance care planning (ACP). This case study illustrates the difficulties involved.

Case summary MH had advanced Multi System Atrophy, a terminal disease involving progressive paralysis and pain and was being cared for at a nursing home. At the time of referral to the Respecting Patient Choices (RPC) Program, she was actively seeking advice from voluntary euthanasia organisations, and was exhibiting increasingly severe symptoms of depression as her disease progressed. The psychiatric liaison service viewed the initiation of involuntary treatment as inappropriate in this context. At this point a referral to the RPC program was made. ACP was conducted with $\mathrm{MH}$, and she and her family completed an ACP including the appointment of a substitute decision maker, and the completion of a refusal of treatment certificate. MH was subsequently admitted to a specialist palliative care unit, where she died shortly after admission.

Discussion This case touches on a number of issues around requests for hastened death, demonstrates a failure of terminal care provision and highlights the need for ACP at the time of diagnosis or early in the course of a terminal illness. Even at a late stage, ACP provided $\mathrm{MH}$ with information regarding her treatment options and allowed her to gain a sense of control over aspects of her treatment.

Case summary MH had been diagnosed with Multi System Atrophy, a terminal disease involving progressive paralysis and pain, 6 years prior to referral and was being cared for at a nursing home. At the time of referral to the RPC Program, Austin Health, Melbourne, she was suicidal, was actively seeking advice from voluntary euthanasia organisations, and had been assessed by the psychiatric liaison service as meeting the diagnostic criteria for major depression. The psychiatric consensus was against initiating involuntary treatment. At this point a referral to the RPC program was made. An ACP Clinician met with $\mathrm{MH}$ and her family to assist her to complete an advance care plan including the appointment of a substitute decision maker and the completion of a refusal of treatment certificate. She was also informed of her right to refuse further medical treatment. MH was subsequently referred, and admitted, to a specialist palliative care unit, where she died within $24 \mathrm{~h}$ of admission.

Discussion This case demonstrates a failure of terminal care provision and highlights the need for ACP at the time of 
diagnosis. Even at a late stage ACP offered an alternative to euthanasia in a situation where she and her family felt that they had no other options. 